\title{
What is Mass and it's Conservation Law
}

\section{Abraham Tamir * \\ Department of Chemical Engineering, Ben-Gurion University of the Negev, Beer-Sheva, Israel}

The oldest theory about the creation of mass is described in Genesis 1:1: "In the beginning God created the heavens and earth", namely, mass was the first thing created. The modern theory of the formation of mass is related to the Big Bang Theory, which is a broadly accepted model for the origin and evolution of our universe. It postulates that the observable universe started from an instantaneously expanding point, roughly 13 to 14 billion years ago, which is also considered as the beginning of time. Since the Big Bang, the universe is continuously expanding, where it is accepted that mass was created after three seconds, galaxies were formed after about five milliards years where man was created after about fifteen milliards years. The creation of
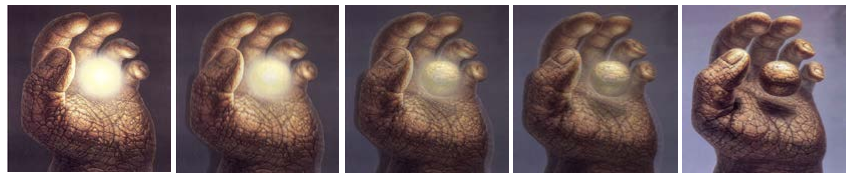

Figure 1: The Big Bang, Formation of mass after 5 seconds, $E=C^{2} \times m$.

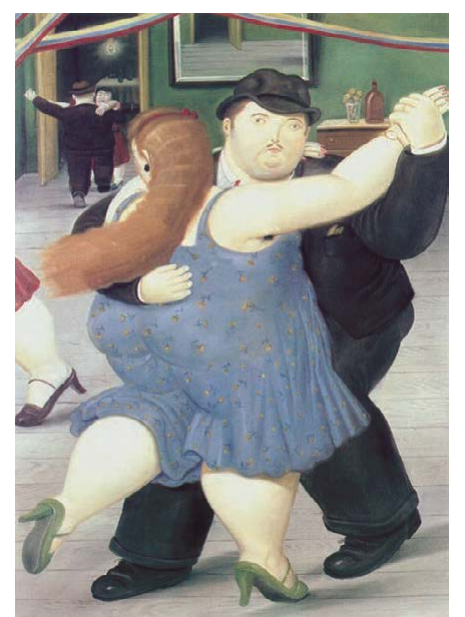

Figure 2: Demonstration of "Inertial mass"

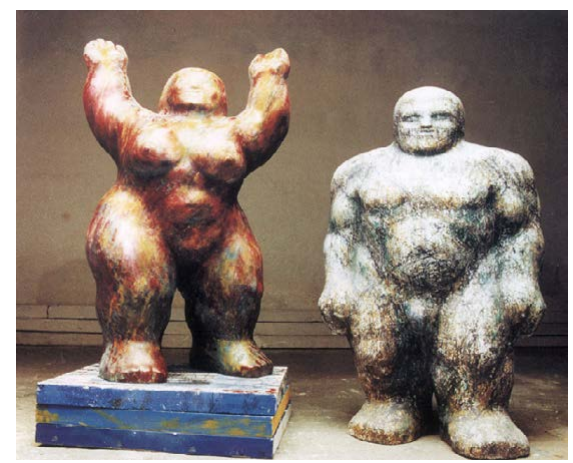

Figure 3: Demonstration of "gravity mass". mass is demonstrated in Figure 1 by five artworks of which only the first and the fifth ones entitled "The Grips" were painted in 1976 by De Es Schwertberger (1942) born in Austria and considered as a mystery artist. The first artwork gives an impression of the Big Bang where the last one of mass. Applying the Photoshop software made it possible to generate artistic simulation in five steps of the creation of mass.

What is mass? It is a physical measure of the amount of substance in a body. It was Isaac Newton (1643-1727) who at about 1687 provided a working definitions for mass according to $m=\rho \mathrm{V}$. However, the Austrian philosopher and physicist Ernst Mach (1838-1916) and other scientists preferred to define mass from Newton's second law $\mathrm{m}=\mathrm{F} / \mathrm{a}$ where force is either attraction or push while its source may be gravitation, electricity, magnetism, or the effort of muscles. This definition is a measure of the inertia of a body and named "inertial mass" because it specifies the resistance of a body to changes in its motion. On the other hand "gravity mass" is defined by the force acting between two bodies according to Newton's General Law of Gravity, F $=\mathrm{m}_{1} \mathrm{~m}_{2} / \mathrm{r}^{2}$. Figure 2, "Dancers", painted in 1987 by Fernando Botero (1932), Colombian, painting voluptuous figures, may describe inertial mass associated with a considerable amount of substance in the two dancers moving in a circular motion. On the other hand, Figure 3, "The Planetarians" sculpture (1987-1991) by Schwertberger demonstrates "gravity mass".

The law of conservation of mass is usually attributed to Antoine Laurent Lavoisier (1743-1794), a French chemist, who established the law in 1789. He was one of the few chemists of his time to fully appreciate the importance that the weight of the products of a chemical reaction must equal the weight of the reactants, which is identical with the following statements of the law, namely, "in any change of state, the total mass is conserved" or "mass is neither created nor destroyed in any chemical reaction." It is interesting to mention that although the paternity of the law of the conservation of mass is generally attributed to Lavoisier, it was known well long before him. It goes back to the ancients Greeks. Anaxagoras expressed it this way in 450 B.C.E.: "Nothing is born or perishes, but already existing things combine, and then separate anew." The law of mass conservation is demonstrated in Figure 4. It is based on Fernando Botero's original painting "Man and Woman" (1989) on the left hand side. The demonstration of the law is based on the version that "in any change of state the total mass is conserved" (except in nuclear reactions) so by combining two halves of the images in different ways the law is demonstrated.

${ }^{*}$ Corresponding author: Abraham Tamir, Department of Chemical Engineering, Ben-Gurion University of the Negev, Beer-Sheva, Israel, Tel: 086472916; E-mail: atamir4@012.net.il

Received September 30, 2014; Accepted October 06, 2014; Published October 13, 2014

Citation: Tamir A (2014) What is Mass and it's Conservation Law. Ind Eng Manage 3: 139. doi: 10.4172/2169-0316.1000139

Copyright: (c) 2014 Tamir A. This is an open-access article distributed under the terms of the Creative Commons Attribution License, which permits unrestricted use, distribution, and reproduction in any medium, provided the original author and source are credited. 


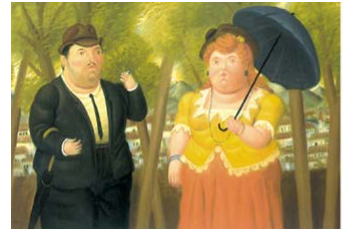

$\mathrm{m}_{1}+\mathrm{m}_{2}$

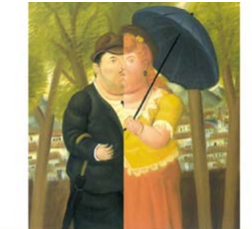

$=\quad 0.5 m_{1}+0.5 m_{2}$

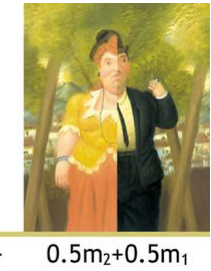

Figure 4: The Law of Mass Conservation.

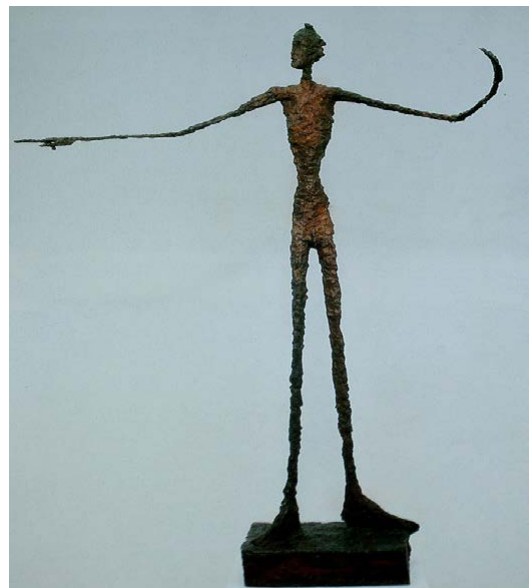

Figure 5: m approaches zero.

About two hundred years scientists believed that Newton's approach was eternal, which assumed that mass was independent of its motion. And then came Einstein, and at the beginning of the twenty century

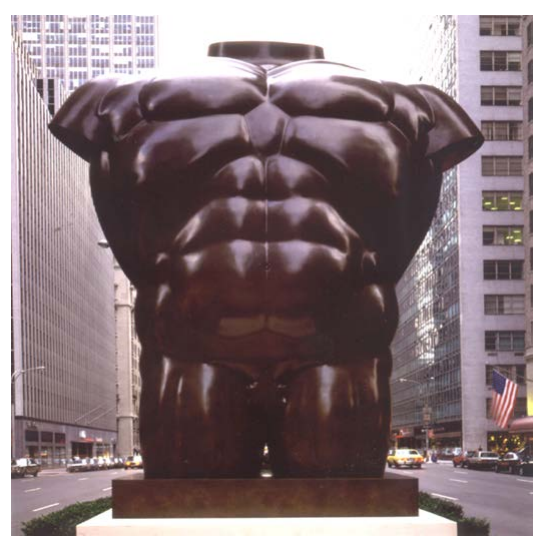

Figure 6: $\mathrm{m}$ approaches infinity $\mathrm{m}$ approaches infinity.

presented his special relativity theory which led to the conclusion among others that the mass of a body increases with its speed according to $\mathrm{m}=\mathrm{m}_{\mathrm{o}}\left[1-(\mathrm{v} / \mathrm{c})^{2}\right]^{0.5}$ where $\mathrm{m}_{\mathrm{o}}$ is the mass of the body at rest, $\mathrm{m}$ is its mass when it is moving with a speed $\mathrm{v}$ relative to an observer where $\mathrm{c}$ is the speed of light. In addition Einstein concluded that mass and energy are interrelated by $\mathrm{E}=\mathrm{mc}^{2}$. In the following, the above results are demonstrated through art. Figure 5, entitled "Man Pointing" (1947) by Alberto Giacometti (1901-1966) Swiss Surrealist painter and sculptor, demonstrates a mass approaching zero. This is by comparison to Figure 6 entitled "Male Torso" (1992) demonstrating mass approaching to infinity, a sculpture by Fernando Botero. Figure 1 may demonstrate the equivalence between mass and energy via art. The left hand side artwork gives an impression of energy whereas the right hand side artwork illustrates mass, both painted by Schwertberger. In conclusion it is expected that the concept of "mass", the most encountered thing in our universe, becomes clearer following its demonstration through art. 